\title{
Anomalous Origin of the Left Pulmonary Artery from the Ascending Aorta. Successful Surgical Correction in an Infant with Fallot's Tetralogy
}

\author{
Fernando Amaral, Mônica A. C. Teixeira, João A. Granzotti, Paulo Henrique Manso, Walter V. A. Vicente
}

Ribeirão Preto, SP - Brazil

We describe the case of a 40-day-old female patient with a history of breathlessness since birth who was referred to our hospital for surgical correction of common arterial trunk. The invasive investigation disclosed a Fallotфs tetralogy anatomy associated with an anomalous origin of the left pulmonary artery from the ascending aorta. Immediately after diagnosis, the patient underwent a successful total surgical correction of the defect, including simultaneous anastomosis of the left pulmonary artery to the pulmonary trunk.

Fallot's tetralogy (FT) is a frequently encountered congenital heart defect, and the detailed diagnostic criteria are well established. However, some discussion still occurs regarding treatment, particularly when surgical correction is contemplated for an infant. Other lesions might be associated with FT; among them, the anomalous origin of the left pulmonary artery (LPA) from the ascending aorta is a rare one. It is well know that when occurring as an isolated lesion, the right pulmonary artery branch is usually involved and when associated with FT, the left branch is more commonly affected. The diagnosis of this anomaly, which may be mistaken for absence of the LPA, should be done soon in life to avoid unilateral pulmonary hypertension and also for appropriate surgical planning. We present the case of a 40day-old patient with the diagnosis of FT and associated anomalous origin of the LPA from the ascending aorta.

\section{Case Report}

A 40-day-old female patient, born in Ituverava, SP, with a history of breathlessness when feeding was referred

Hospital das Clínicas da Faculdade de Medicina de Ribeirão Preto - USP e Faculdade de Medicina da UNAERP - Ribeirão Preto

Mailing address: Fernando Amaral - Av. Independência, 1379 - 14025-390 Ribeirão Preto, SP - Brazil - E-mail: ftvamaral@bol.com to the Hospital das Clínicas da Faculdade de Medicina de Ribeirão Preto for surgical correction of a common arterial trunk. On admission, the patient was pink and breathing normally. The weight was $2,200 \mathrm{~kg}$ and the arterial oxygen saturation was $94 \%$ at room air. During auscultation, an ejection systolic murmur +++/6 was best heard in the pulmonary area with a single second sound. The arterial pulses were normal and heart rate was $130 \mathrm{bpm}$. The electrocardiogram showed signs of biventricular hypertrophy (fig. 1), and in the chest X-ray, an obvious heart enlargement was seen (fig. 2). The 2D echocardiogram showed the features of a classic FT associated with a possible anomalous vessel originating from the ascending aorta. Based on this information, the infant underwent cardiac catheterization that confirmed the Fallot $\notin$ s tetralogy anatomy with a hypoplastic pulmonary trunk and anomalous origin of the LPA from the ascending aorta (fig. 3). The systolic pressure in both ventricles was $68 \mathrm{mmHg}$, and 25 days after admission the patient weighing $2,900 \mathrm{~kg}$ was referred for correction of the defects.

The thymus gland was noted to be absent. Initially, careful dissection of the ascending aorta, pulmonary artery, and left pulmonary artery was undertaken. Under hypothermic bypass and blood cardioplegia, the $12-\mathrm{mm}$ ventricular septal defect was closed with a Dacron patch through the tricuspid valve. Two commissural plications were necessary to achieve a competent tricuspid valve. The left pulmonary artery was detached from the aorta and the aortotomy closed in 2 planes. Pulmonary transanular plasty was done with a piece of autologous pericardium, leaving the valve ring adequate for the $5-\mathrm{mm}$ Hegar dilator (expected caliber for the patient's body surface area). The procedure was completed by connecting the left pulmonary artery to the lateral surface of the pulmonary trunk and atrial closure. Coming off bypass was uneventful and the right ventricular/aorta pressure rate was 0.59 . The postoperative period was marked by moderate cardiac failure and coagulation disturbances, both of them controlled pharmacologically, and also by surgical scar infection requiring reinter- 


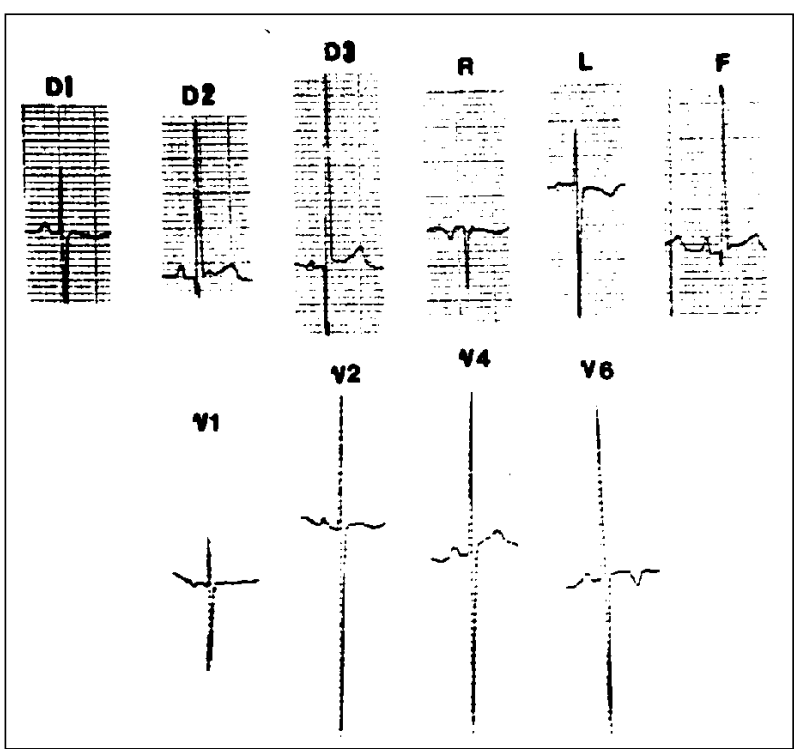

Fig. 1 - 12-lead electrocardiogram showing signs of biventricular hypertrophy.

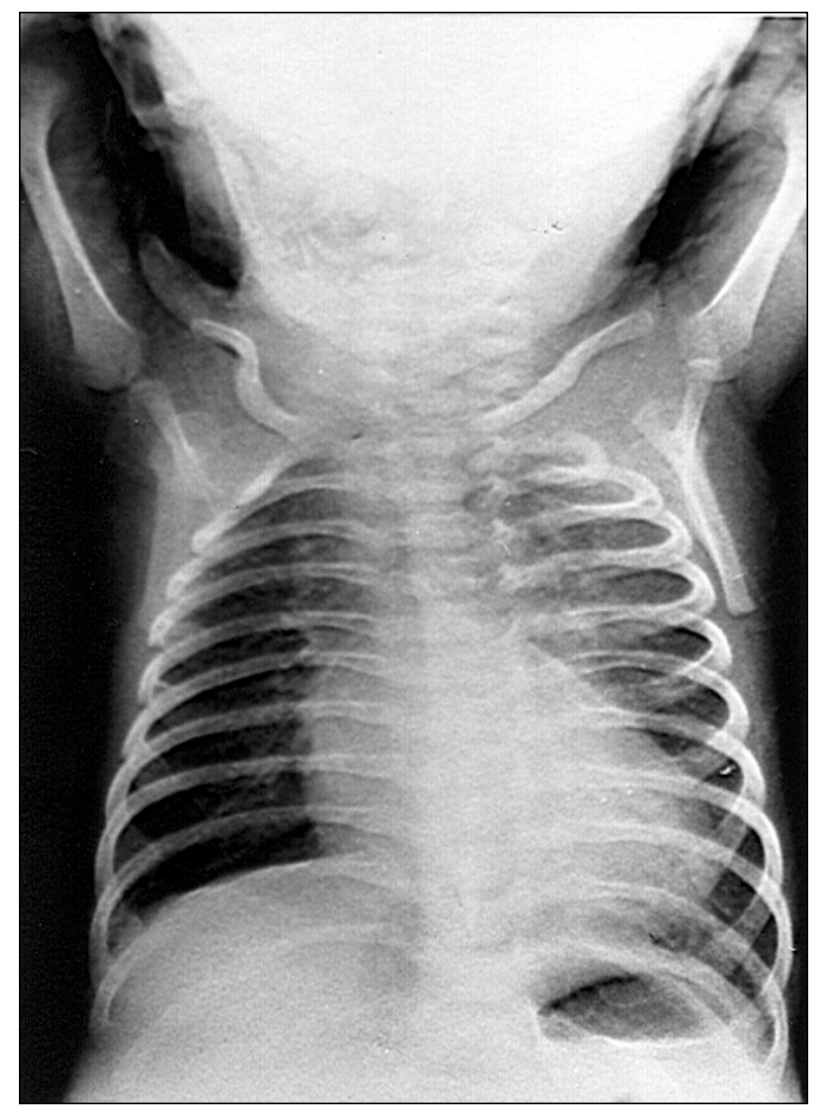

Fig. 2 - Chest X-ray disclosing an enlarged heart.

vention in the 9th postoperative day, which kept the child for 18 days in the intensive care unit. Ten months after the operation the child is well, weighing $6,650 \mathrm{~kg}$, on no medication, and with a residual systolic murmur in the pulmonary area. The electrocardiogram recently recorded showed complete right bundle- branch block, and in the echocardiogram signs were present of residual pulmonary stenosis and

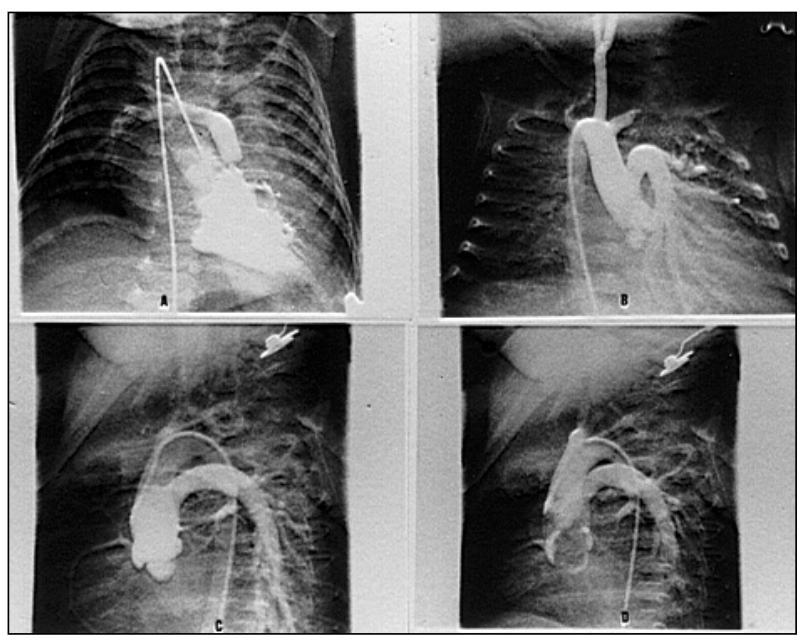

Fig. 3 - Hemodynamic and angiocardiographic investigation. Right ventriculography in frontal plane (A) disclosing severe infundibular obstruction and isolated connection of the right ventricle to the right pulmonary artery. Aortography in frontal (B) and lateral (C, D) planes disclosing an anomalous connection between the left pulmonary artery and the ascending aorta.

regurgitation with an adequate connection between the pulmonary trunk and the LPA.

\section{Discussion}

First described by Thomas in $1960^{1}$, anomalous origin of the pulmonary artery (AOPA) is a very rare entity, usually associated with $\mathrm{FT}^{2}$. Clinical suspicion is difficult, despite some reports mentioning a plethoric left lung. The diagnosis is usually suspected on the echocardiogram, as here reported, and the first clue is an apparent absence of the LPA. The final diagnosis is, however, made by cardiac catheterization, disclosing an isolated connection between the right ventricle and the right pulmonary artery after right ventriculography, without opacification of the LPA, which can be visualized through aortography. It is important to emphasize that the early diagnosis of this anomaly is critical in the long-term follow-up of these patients because of the possible development of pulmonary hypertension. Another considerable risk is the interpretation of the echo or hemodynamic findings or both as an apparent absence of the LPA, which might occur in FT, without searching for its aortic origin. Based on these possibilities, we think that in cases of FT with a nonvisualized LPA, its aortic origin should be searched for.

Soon after the first description of the AOPA, 4 decades ago, little information was available. By 1973, 20 cases had been described, among them only 3 treated by operation ${ }^{3}$. After that, other reports appeared, emphasizing the possibility of surgical correction ${ }^{4}$, including patients with associated Di George syndrome ${ }^{5}$. In 1989, 6 cases have been described in detail by Fong et $\mathrm{al}^{6}$, two of them associated with FT. It should be mentioned that two of these patients, with an approximate age of 2 years, died soon after operation, and in the autopsy left pulmonary hypertension was evident.

The case here presented, the first in Brazil as far as we know, despite the good national experience with FT, des- 
cribes a 40-day-old infant with a history of breathlessness and heart enlargement secondary to an increased lung flow due to a left to right shunt from the ascending aorta to the LPA. The diagnosis was suspected on the echocardiogram and the anomaly confirmed by cardiac catheterization. Nowadays, the surgical correction of FT is usually made between the 6th and 12th month of life in the majority of the reference centers. However, in the case above described the procedure was indicated early due to the increased risk of developing pulmonary hypertension.

In conclusion, we believe that AOPA is a very rare entity, which should be remembered in every patient under investigation with a clinical diagnosis of FT, particularly in cases where the LPA appears to be "absent." Surgical correction should be done soon after diagnosis if pulmonary hypertension is to be avoided. The neonatal repair is feasible and should be discussed, as recently reported in a case of anomalous origin of the right pulmonary artery from the ascending aorta ${ }^{7}$. Despite the small experience registered, due to the rarity of the disease, a good prognosis can be anticipated for the cases operated on soon, based on the acquired experience with FT.

\section{References}

1. Thomas HW. Cardiovascular anomalies, congenital cardiac malformations. J Tech Methods 1960; 58: 21-9.

2. Tagliente MR, Troise D, Milela L, Vairo H. Isolated anomalous origin of left pulmonary artery from the ascending aorta. Am Heart J 1996; 132: 1289-92.

3. Imai Y, Nishiya Y, Morikawa T, Kurosawa H, Konno S. Total correction of tetralogy of Fallot associated with an anomalous left pulmonary artery arising from the aortic arch. J Thorac Cardiovasc Surg 1974; 68: 51-8.

4. Robin E, Silberberg B, Ganguly SN, Magnisalis K. Aortic origin of the left pulmonary artery. Variant of tetralogy of Fallot. Am J Cardiol 1975; 35: 324-9.

5. Dodo H, Alejos JC, Perloff JK, Lars H, Drinkwater DC, Williams RG. Anoma- lous origin of the left pulmonary artery from the ascending aorta associated with Di George syndrome. Am J Cardiol 1995; 75: 1294-5.

6. Fong LV, Anderson RH, Siewers RD, Trento A, Park SC. Anomalous origin of one pulmonary artery from the ascending aorta: a review of echocardiographic, catheter and morphological features. Br Heart J 1989; 62: 389-95.

7. Miyazaki K, Murashita T, Kubota T, Matsuzaki K, Miyazaki YJ, Yasuda K. Neonatal repair of anomalous origin of the right pulmonary artery from the ascending aorta: a case report and review in the literature. J Cardiovasc Surg $2000 ; 41: 863-8$ 